\title{
Gallinaza y aserrín descompuesto y su efecto en el rendimiento del ají charapita en Aguaytía, Ucayali
}

\section{Chicken and sawdust decomposed and their effect on the performance of chili pepper in Aguaytía, Ucayali}

\author{
Hammerly Freddy López Martín ${ }^{1}$, Pablo Pedro Villegas Panduro ${ }^{1}$, Diana Prince Zumaeta Sangama de \\ Villegas $^{1}$, Roger Brayan Braga Sandoval ${ }^{1}$ y Carmen del Pilar Espinoza Pérez ${ }^{1}$
}

\begin{abstract}
${ }^{1}$ Universidad Nacional de Ucayali - Perú. Carretera Federico Basadre Km 5.5, Callería. Ucayali. Facultad de Ciencias Agropecuarias, e-mail: hammerlylm@gmail.com

${ }^{1}$ Universidad Nacional de Ucayali - Perú. Carretera Federico Basadre Km 5.5, Callería. Ucayali. Facultad de Ciencias Agropecuarias, e-mail: ppvillegasunu@gmail.com, ORCID: https://orcid.org/0000-0001-9300-8113.

${ }^{1}$ Universidad Nacional de Ucayali - Perú. Carretera Federico Basadre Km 5.5, Callería. Ucayali. Facultad de Ciencias Agropecuarias, e-mail: dianaprins19@hotmail.com

${ }^{1}$ Universidad Nacional de Ucayali - Perú. Carretera Federico Basadre Km 5.5, Callería. Ucayali. Facultad de Ciencias Agropecuarias, e-mail: roger_braga@unu.edu.pe ORCID: https://orcid.org/0000-0002-5794-1587

${ }^{1}$ Universidad Nacional de Ucayali - Perú. Carretera Federico Basadre Km 5.5, Callería. Ucayali. Facultad de Ciencias Agropecuarias, e-mail: carmen_espinoza@unu.edu.pe
\end{abstract}

\section{Resumen}

Se estudió el efecto de la aplicación de tres niveles de gallinaza y tres niveles de aserrín descompuesto en el rendimiento del ají charapita (Capsicum frutescens L.) en Aguaytía Ucayali, ubicado en el Km. 171 de la Carretera Federico Basadre margen izquierda, Pucallpa - Lima, interior 3,2 Km, para los cuales, se elaboró un vivero para el almacigado y en repique de las plántulas a bolsas de repique, los cuales posteriormente fueron instalados en el campo de cultivo preparado, al cual se aplicó los abonos orgánicos de manera localizada, al momento de la instalación y cada tres meses, estudiándose dos factores: gallinaza $(0,1$ y $2 \mathrm{~kg} /$ planta) y aserrín descompuesto (0, 1 y 2 $\mathrm{kg} /$ planta) y sus combinaciones, bajo un diseño de bloque completo al azar con un arreglo factorial 3 x 3 y una prueba de promedios de Duncan al 0,05\%, determinándose que, a medida que se aumenta la cantidad de gallinaza descompuesta aplicada a la planta, aumentan los valores en las variables de desarrollo vegetativo y de rendimiento, siendo la aplicación de $6 \mathrm{~kg}$ de gallinaza por planta la que logró los mejores promedios en todas las variables estudiadas, observándose además que, a medida que se aumenta la cantidad de aserrín descompuesto aplicada a la planta, los valores en todas las variables estudiadas disminuyen, siendo el tratamiento sin aplicación de aserrín descompuesto el que logró los mejores promedios, corroborándose este comportamiento en la interacción de los factores, en los cuales la aplicación de $6 \mathrm{~kg}$ de gallinaza descompuesta sola logró los mejores promedios en todas las variables estudiadas.

Palabras clave: Capsicum frutescens L., vivero, desarrollo vegetativo, rendimiento.

\begin{tabular}{l} 
Abstract \\
The effect of the application of three levels of chicken manure and three levels of decomposed \\
sawdust on the yield of the chili pepper (Capsicum frutescens L.) in Aguaytía - Ucayali, located at \\
Km. 171 of the Carretera Federico Basadre left bank, Pucallpa was studied. - Lima, interior $3.2 \mathrm{~km}$, \\
for which, a nursery was made for storing and ringing the seedlings into ringing bags, which were \\
\hline Carretera Federico Basadre Km 6 , Dirección de Producción Intelectual \\
Downloadable from: 664
\end{tabular}

Revista de Investigación Universitaria por Universidad Nacional de Ucayali se distribuye bajo una Licencia Creative Commons Atribución-NoComercial 4.0 Internacional. 
later installed in the prepared crop field, to which the organic fertilizers were applied. in a localized manner, at the time of installation and every three months, studying two factors: chicken manure ( 0 , 1 and $2 \mathrm{~kg} /$ plant) and decomposed sawdust (0,1 and $2 \mathrm{~kg} / \mathrm{plant})$ and their combinations, under a design of complete random block with a $3 \times 3$ factorial arrangement and a Duncan mean test at $0.05 \%$, determining that, as the amount of decomposed manure applied to the plant increases, the values in the development variables increase vegetative o and yield, being the application of $6 \mathrm{~kg}$ of chicken manure per plant which achieved the best averages in all the variables studied, also observing that, as the amount of decomposed sawdust applied to the plant is increased, the values in all the The variables studied decrease, being the treatment without the application of decomposed sawdust the one that achieved the best averages, corroborating this behavior in the interaction of the factors, in which the application of $6 \mathrm{~kg}$ of decomposed chicken manure alone achieved the best averages in all the variables studied.

Keywords: Capsicum frutescens L., nursery, vegetative development, yield

\section{Introducción}

Capsicum frutescens se caracteriza por sus variedades muy picantes de ajíes, con un origen no determinado, pero principalmente cultivado en Centro y Sur América. En el Perú se han descubierto restos arqueológicos de esta especie, con una antigüedad de $1200 \mathrm{~A}$. C., siendo el ají charapita, una de las variedades más representativas, considerándose uno de los ajíes nativos más picantes, con un alto consumo en la región de la selva, siendo este ají, el primer ají de la selva en ser exportado y con gran aceptación en los mercados internacionales (García, 2011). Las variedades de ají de nuestra amazonia más conocidos son: el ají charapita (Capsicum frutescens), el ají dulce (Capsicum chínense), el ají ayucllo (Capsicum baccatum var. Pendulum), el ají pipi de mono (Capsicum frutescens) entre otros, siendo el

\section{5}

Downloadable from: http://revistas.unu.edu.pe

Carretera Federico Basadre Km 6, Dirección de Producción Intelectual más conocido y de mayor consumo, el ají charapita (Garcia Yi, 2013)

Miranda y Rengifo (2016) estudiaron las dosis aplicación de guano de isla y compost $(0.5$ y 1 kg) en plantas de ají charapita bajo condiciones de clima y suelo del distrito de Manantay, Pucallpa, a un distanciamiento de $1 \mathrm{~m} \quad \mathrm{x} 1 \mathrm{~m}$ para cada tratamiento, demostrándose que no existen diferencias significativas entre los tratamientos estudiados, optándose por utilizar las dosis de $0.5 \mathrm{~kg}$ de guano de isla y compost por el costo.

Velásquez (2016) estudio el efecto de fertilizantes foliares orgánico sobre el rendimiento de ají Amarillo (Capsicum baccatum L.var pendulum) en el Valle de Mala (Perú), demostrándose que no hubieron diferencias significativas en el rendimiento, sin embargo, las plantas fertilizadas como el

\section{(c) (i) \&)}

Revista de Investigación Universitaria por Universidad Nacional de Ucayali se distribuye bajo una Licencia Creative Commons Atribución-NoComercial 4.0 Internacional. 
biol derivado de rastrojos de un campo orgánico al 30\% logró 9,086 t/ha y el biol derivado de la industria de harina de pescado al $0.5 \%$, logro 8,915 t/ha, en comparación al testigo el cual logró 6,853.30 t/ha. Masaquiza (2016) estudio la respuesta de dos híbridos de pimiento "Nathalie" y "Martha", a tres tratamientos de biol elaborados con gallinaza, estiércol de cerdo y vacuno respectivamente, sobre las características agronómicas del cultivo, demostrándose que los bioles a base de gallinaza y estiércol de cerdo mostraron mayor rendimiento de frutos. Ocupa (2019) estudio el efecto de tres dosis de biol artesanal $(0 \%, 5 \%$ y $10 \%)$, en el rendimiento y peso de fruto en el cultivo de ají páprika morrón en Marcavelica, Sullana, Piura, Perú, determinándose que la aplicación de 10\% de biol logró un rendimiento de $8300 \mathrm{~kg} / \mathrm{ha}$ y un peso de fruto de $20.75 \mathrm{~g}$.

Villalobos (2015) investigó el efecto de cuatro dosis $(1000,800,600,400 \mathrm{~kg} / \mathrm{ha}) \mathrm{de}$ fertilizante orgánico enriquecido con microorganismos eficaces (Ferti EM) en el cultivo de ají pimentón (Capsicum annuum L.) variedad California Wonder, en Lamas, San Martín, observándose los mejores resultados en la aplicación de $1000 \mathrm{~kg} / \mathrm{ha}$ Ferti EM, con $21338,72 \mathrm{~kg} / \mathrm{ha}$ de rendimiento, $185,7 \mathrm{~g}$ de peso del fruto; $8,9 \mathrm{~cm}$ de largo con 7,79 cm diámetro del fruto.

Reyes et al. (2017) compararon la aplicación de abonos orgánicos respecto a una aplicación de fertilización química convencional, en el rendimiento del pimiento (Capsicum annum L.), determinándose que la aplicación de humus de lombriz, y humus de lombriz + Jacinto de agua tuvieron respuestas significativamente mejores respecto al largo, diámetro y peso de los frutos. Lozano (2016) estudio el efecto de cuatro dosis de materia orgánica (sin aplicación, 20, 30 y 40 t/ha de pollaza) en el cultivo de ají pimentón (Capsicum annuum L.) variedad California Wonder, en el distrito de Lamas, demostrándose que la aplicación de 40 t/ha de pollaza logró el mayor rendimiento promedio, con 41120. $7 \mathrm{~kg} / \mathrm{ha} \quad \mathrm{y}$ las mejores características agronómicas en altura de planta con $44.7 \mathrm{~cm}, 24.53$ flores por planta, 19.0 frutos por planta, $8.9 \mathrm{~cm}$ de diámetro del fruto y $246.7 \mathrm{~g}$ de peso del fruto.

Abreu et al. (2018) evaluaron el efecto de diferentes combinaciones de fertilizantes químicos y humus de lombriz (fertilizante químico 10-20-10 y humus de lombriz de estiércol vacuno), sobre el crecimiento y desarrollo de Capsicum annuиm L. cv. 
Magistral, demostrándose que el rendimiento obtenido en la primera cosecha fue superior con la aplicación de fertilizante químico, pero en la segunda cosecha fue similar al tratamiento fertilizante químico $75 \%$ + humus 4, 6 t/ha, evidenciando el potencial del humus de lombriz para reducir el uso de fertilizante químico sin afectar el rendimiento. Cabaleiro et al. (2017) estudiaron la producción y calidad de pimiento tipo Lamuyo en invernadero abonado con estiércol de pollo deshidratado y granulado (BL), demostrándose que el abonado, tanto mineral como orgánico, no originó diferencias significativas en el contenido de vitamina $\mathrm{C}$, asimismo, los suelos abonados con el estiércol de pollo, no presentaron signos de salinización.

En la investigación se evaluó el efecto de la aplicación de tres niveles de gallinaza y tres niveles de aserrín descompuesto en el rendimiento del ají charapita (Capsicum frutescens L.) en Aguaytía - Ucayali.

\section{Métodos}

\section{Ubicación y evaluación de suelos del campo experimental}

La investigación se desarrolló en el fundo "El Gato", ubicado en el Km. 171 de la Carretera Federico Basadre, margen izquierda carretera Pucallpa - Lima, interior 3,2 Km, ubicado a $09^{\circ} 04^{\prime} 45,9$ " Latitud Sur y $75^{\circ} 35^{\prime} 20,4$ " Longitud Oeste.

Se extrajo muestras a 0-25 $\mathrm{cm}$ de profundidad de suelo del área a emplearse, usando el método del zig - zag, para conformar un kilo de muestra en cada bloque, los cuales fueron analizados en el laboratorio de suelos de la UNU.

Tabla 1

Resultados del análisis de suelo del campo experimental de ají charapita.

\begin{tabular}{|c|c|c|c|c|c|c|c|c|c|c|c|}
\hline \multirow{2}{*}{$\begin{array}{l}\text { Bloque } \\
\text { (prof.: } 0 \\
-20 \mathrm{~cm} \text { ) }\end{array}$} & \multirow{2}{*}{$\begin{array}{c}\% \\
\text { arena }\end{array}$} & \multirow{2}{*}{$\begin{array}{c}\% \\
\text { arcilla }\end{array}$} & \multirow{2}{*}{$\begin{array}{c}\% \\
\text { limo }\end{array}$} & \multirow{2}{*}{$\begin{array}{c}\text { Clase } \\
\text { textural }\end{array}$} & \multirow[b]{2}{*}{$\mathbf{p H}$} & \multirow{2}{*}{$\begin{array}{c}\mathbf{P} \\
(\mathbf{p p m})\end{array}$} & \multicolumn{3}{|c|}{ CIC (Meq/100 g) } & \multirow{2}{*}{$\underset{\%}{\text { MO }}$} & \multirow{2}{*}{$\begin{array}{c}\mathrm{N} \\
\text { total } \\
\%\end{array}$} \\
\hline & & & & & & & $\mathbf{K}$ & Mg & $\mathbf{C a}$ & & \\
\hline I & 81,00 & 12,28 & 6,72 & $\begin{array}{c}\text { Franco } \\
\text { arenoso }\end{array}$ & $\begin{array}{c}6,6 \\
3\end{array}$ & 3,74 & 0,2 & 0,71 & 0,65 & 2,8 & 1,12 \\
\hline II & 77,4 & 13,88 & 8,72 & $\begin{array}{c}\text { Franco } \\
\text { arenoso }\end{array}$ & $\begin{array}{c}6,6 \\
4\end{array}$ & 2,69 & 0,3 & 0,85 & 0,40 & 0,55 & 0,22 \\
\hline III & 75,4 & 14,28 & $\begin{array}{c}10,3 \\
2\end{array}$ & $\begin{array}{c}\text { Franco } \\
\text { arenoso }\end{array}$ & $\begin{array}{c}6,5 \\
4\end{array}$ & 2,43 & 0,2 & 0,90 & 0,50 & 2,03 & 0,81 \\
\hline
\end{tabular}

Fuente: Laboratorio de Suelos - UNU 
Se seleccionaron plantas madres en periodo de producción, de los cuales se seleccionaron frutos con buenas características y que hayan cumplido su madurez fisiológica, para luego extraer las semillas, enjuagarlas y colocarlas en papal, bajo sombra para su secado por 24 horas.

\section{Almacigado, instalación y aplicación de los abonos orgánicos}

Se construyó un vivero empleándose madera rolliza y techo de hojas de palma de la zona, y en el interior del vivero se acondiciono una cama almaciguera con arena de rio como sustrato en el cual se sembraron las semillas tratadas con fungicida Carboxín + Captan, polvo mojable, las cuales germinaron a los 10 días, y se mantuvo hasta que las plántulas alcanzaron los $10 \mathrm{~cm}$ de altura.

El sustrato se preparó con 2 partes de materia orgánica, 1 parte de suelo limoso y 1 parte de arena, con el cual se llenó las bolsas de repique de 5 x 7 x 1.5 pulgadas, en los cuales se realizó el repique de las plántulas.

Se colectó gallinaza y aserrín de las granjas y de los aserraderos locales de Aguaytía, y se procedió a descomponerlos, formando montículos y regando diariamente, por un espacio de 40 días. Luego se extrajo 1 kilo de muestra de cada abono su análisis correspondiente.

Tabla 2

Resultados del análisis de la gallinaza y el aserrín descompuesto utilizado en el trabajo experimental

\begin{tabular}{lccccc}
\hline \multicolumn{1}{c}{ Fertilizante } & $\mathbf{p H}$ & $\mathbf{P}(\mathbf{p p m})$ & Acidez & Mg (Meq) & Ca (Meq) \\
\hline Gallinaza descompuesta & 5,84 & 61,00 & 3,1 & 1,95 & 0,60 \\
Aserrín descompuesto & 5,66 & 6,95 & 2,0 & 1,45 & 0,94 \\
\hline
\end{tabular}

Fuente: Laboratorio de Suelos - UNU

Cuando las plantas lograron una altura de 15 $\mathrm{cm}$, se les llevó a campo definitivo, preparando hoyos de $30 \mathrm{~cm}$ de largo x $30 \mathrm{~cm}$ de ancho $\mathrm{x}$ $30 \mathrm{~cm}$ de profundidad, en la que se aplicó los tratamientos de abonos orgánicos en estudio, utilizándose un distanciamiento de $1 \mathrm{~m}$ x 1,5 $\mathrm{m}$.

La aplicación de los abonos se realizó al momento de la instalación en campo y posteriormente se realizó los anillos de 
abonamiento cada 3 meses, hasta la culminación del estudio.

\section{Análisis estadístico}

Se aplicó el diseño de bloques completamente al azar, con arreglo factorial, con dos factores A: Gallinaza descompuesta con 03 niveles ( 0 $\mathrm{kg} /$ planta, $3 \mathrm{~kg} / \mathrm{planta}$ y $6 \mathrm{~kg} /$ planta) y el factor B: Aserrín descompuesto con 03 niveles (0 kg/planta, 3 kg/planta y 6 kg/planta). Se utilizó la prueba de Duncan al 0,05\% de significancia.

\section{Resultados y discusión}

Variables de desarrollo vegetativo y rendimiento para el factor gallinaza descompuesta

La tabla 3 muestra la prueba de promedios de Duncan para las variables de desarrollo vegetativo (altura de planta, diámetro de copa, diámetro de tallo) y las variables de rendimiento (longitud de fruto, peso de frutos por planta y rendimiento por hectárea) para la gallinaza descompuesta en el ají charapita a los 183 días de instalados en campo, demostrándose que a medida que se aumenta la cantidad de gallinaza descompuesta aplicada a la planta, aumentan los valores en todas las variables estudiadas, de tal manera que la aplicación de $6 \mathrm{~kg}$ de gallinaza por planta lograron los mejores promedios en todas las variables estudiadas, resaltándose el rendimiento por hectárea logrado por este tratamiento $(28333 \mathrm{~kg} / \mathrm{ha})$. Al respecto Riva (2019) afirma que el rendimiento del ají charapita en el Fundo de la UNU, en Pucallpa el cual es una terraza alta, a un distanciamiento de $0,80 \mathrm{~m}$ x $0,80 \mathrm{~m}$ fue de 489,67 gramos por planta y $7651,17 \mathrm{~kg} / \mathrm{ha}$ y a un distanciamientos de $2 \mathrm{~m} 1 \mathrm{~m}$ fue de 736,76 g/planta y $3683,80 \mathrm{~kg} / \mathrm{ha}$, y para las condiciones del Caserío José Olaya el cual es un suelo aluvial, a un distanciamiento de $1 \mathrm{~m}$ x 1m, fue de $1200 \mathrm{~g} /$ planta y $12000 \mathrm{~kg} / \mathrm{ha}$, siendo estos rendimientos significativamente inferiores a los obtenidos en el estudio en los cuales la aplicación de 6 kg/planta de gallinaza descompuesta logró rendimientos de $4250 \mathrm{~g} /$ planta y $28333 \mathrm{~kg} / \mathrm{ha}$ a un distanciamiento de 1m x 1,5 m. López (2015) estudio la aplicación de biol y gallinaza en la producción del ají tabasco (Capsicum annum L.) en Quevedo Santo Domingo, Tsáchilas, concluyéndose que, la gallinaza tuvo el mejor comportamiento en las variables agronómicas, concordando con los resultados obtenidos en esta investigación. Sin embargo, Arévalo (2012) evaluó el rendimiento de cinco densidades de siembra de Capsicum frutescens L. (Ají Charapita) en un Ultisols de Pucallpa, demostrándose que la densidad de 
siembra de $0.8 \mathrm{~m} \times 0.8 \mathrm{~m}$, logro un rendimiento de $7651.17 \mathrm{~kg} / \mathrm{ha}$, superando a las densidades de siembra de $1 \mathrm{~m}$ x $1.5 \mathrm{~m}, 1 \mathrm{~m}$ x $2 \mathrm{~m}, 1.5 \mathrm{~m}$ x $1.5 \mathrm{~m}$ y $1.5 \mathrm{~m} \times 2 \mathrm{~m}$ los cuales lograron rendimientos de $33393.60 \mathrm{~kg} / \mathrm{ha}, 2437.00$ kg/ha, $2234.96 \mathrm{~kg} / \mathrm{ha} \quad$ y $1582.03 \mathrm{~kg} / \mathrm{ha}$; respectivamente, los cuales son rendimiento muy bajos comparados con los resultados obtenidos en este estudio.

Tabla 3

Variables de desarrollo vegetativo y rendimiento para el factor gallinaza descompuesta, a los 183 días de instalado en campo definitivo

\begin{tabular}{cccccccc}
\hline $\begin{array}{c}\text { Gallinaza } \\
(\mathbf{k g} / \text { planta })\end{array}$ & $\begin{array}{c}\text { Alt. } \\
\text { planta } \\
(\mathbf{c m})\end{array}$ & $\begin{array}{c}\text { Diám. } \\
\mathbf{c o p a} \\
(\mathbf{c m})\end{array}$ & $\begin{array}{c}\text { Diám. } \\
\text { tallo } \\
(\mathbf{c m})\end{array}$ & $\begin{array}{c}\text { Diám. } \\
\text { fruto } \\
(\mathbf{c m})\end{array}$ & $\begin{array}{c}\text { Long. } \\
\text { fruto } \\
(\mathbf{c m})\end{array}$ & $\begin{array}{c}\text { Peso } \\
\text { frutos/ } \\
\text { planta }(\mathbf{k g})\end{array}$ & $\begin{array}{c}\text { Rend. } \\
(\mathbf{k g} / \mathrm{ha})\end{array}$ \\
\hline $0 \mathrm{~kg}$ & $74.25 \mathrm{~b}$ & $90.30 \mathrm{~b}$ & $1.71 \mathrm{~b}$ & $1.22 \mathrm{c}$ & $1.03 \mathrm{c}$ & $2.817 \mathrm{c}$ & $18783 \mathrm{c}$ \\
$3 \mathrm{~kg}$ & $83.94 \mathrm{a}$ & $104.77 \mathrm{a}$ & $1.75 \mathrm{~b}$ & $1.28 \mathrm{~b}$ & $1.08 \mathrm{~b}$ & $3.836 \mathrm{~b}$ & $25574 \mathrm{~b}$ \\
$6 \mathrm{~kg}$ & $89.11 \mathrm{a}$ & $110.27 \mathrm{a}$ & $1.81 \mathrm{a}$ & $1.38 \mathrm{a}$ & $1.17 \mathrm{a}$ & $4.250 \mathrm{a}$ & $28333 \mathrm{a}$ \\
\hline
\end{tabular}

*Letras iguales no presentan diferencias significativas. Duncan $\mathrm{p} \leq 0,05$

Variables de desarrollo vegetativo y rendimiento para el factor aserrín descompuesto

La tabla 4 muestra la prueba de promedios de Duncan para las variables de desarrollo vegetativo (altura de planta, diámetro de copa, diámetro de tallo) y las variables de rendimiento (longitud de fruto, peso de frutos por planta y rendimiento por hectárea) para el aserrín descompuesto en el ají charapita a los 183 días de instalados en campo, demostrándose que a medida que se aumenta la cantidad de aserrín descompuesto aplicada a la planta, los valores en todas las variables estudiadas disminuyen, de tal manera que el tratamiento sin aplicación de aserrín descompuesto logró los mejores promedios en todas las variables estudiadas, resaltándose el rendimiento por hectárea logrado por este tratamiento (26816 kg/ha). Al respecto, Riva (2019) indica que los rendimientos mayores se observan en el ecosistema aluvial (caserío José Olaya con $12000,00 \mathrm{~kg} / \mathrm{ha}$ ), por las características favorables que presentan estos suelos: $\mathrm{pH}$ mayores de 6 y $4.25 \%$ de materia orgánica, sin embargo, a pesar que los rendimientos con aplicación de aserrín descompuesto, son bajos comparado con el testigo, el suelo de aluvial de Aguaytía genero 
rendimientos superiores a los reportados por

Riva (2019). Asimismo, Mozombite (2012) estudio el nivel adecuado de aplicación orgánica (1 kg, 2 kg, 3 kg y 4 kg de humus de lombriz/planta) para incrementar la producción del cultivo de ají charapita (Capsicum frutescens) en Pucallpa, Ucayali,

Tabla 4

Variables de desarrollo vegetativo y rendimiento para el factor aserrín descompuesto, a los 183 días de instalado en campo definitivo.

\begin{tabular}{cccccccc}
\hline $\begin{array}{c}\text { Aserrín } \\
(\mathbf{k g} / \text { planta) }\end{array}$ & $\begin{array}{c}\text { Alt. } \\
\text { planta } \\
(\mathbf{c m})\end{array}$ & $\begin{array}{c}\text { Diám. } \\
\text { copa } \\
(\mathbf{c m})\end{array}$ & $\begin{array}{c}\text { Diám. } \\
\text { tallo } \\
(\mathbf{c m})\end{array}$ & $\begin{array}{c}\text { Diám. } \\
\text { fruto } \\
(\mathbf{c m})\end{array}$ & $\begin{array}{c}\text { Long. } \\
\text { fruto } \\
(\mathbf{c m})\end{array}$ & $\begin{array}{c}\text { Peso frutos/ } \\
\text { planta }(\mathbf{k g})\end{array}$ & $\begin{array}{c}\text { Rend. } \\
(\mathbf{k g} / \mathbf{h a})\end{array}$ \\
\hline $0 \mathrm{~kg}$ & $92.36 \mathrm{a}$ & $114.66 \mathrm{a}$ & $1.82 \mathrm{a}$ & $1.31 \mathrm{a}$ & $1.09 \mathrm{a}$ & $4.022 \mathrm{a}$ & $26816 \mathrm{a}$ \\
$3 \mathrm{~kg}$ & $77.50 \mathrm{~b}$ & $91.58 \mathrm{~b}$ & $1.73 \mathrm{~b}$ & $1.26 \mathrm{~b}$ & $1.08 \mathrm{a}$ & $3.192 \mathrm{~b}$ & $21282 \mathrm{~b}$ \\
$6 \mathrm{~kg}$ & $77.44 \mathrm{~b}$ & $99.11 \mathrm{~b}$ & $1.72 \mathrm{~b}$ & $1.30 \mathrm{a}$ & $1.10 \mathrm{a}$ & $3.688 \mathrm{a}$ & $24593 \mathrm{a}$ \\
\hline
\end{tabular}

*Letras iguales no presentan diferencias significativas. Duncan $\mathrm{p} \leq 0,05$

Variables de desarrollo vegetativo y rendimiento para la interacción entre el factor gallinaza descompuesta y el aserrín descompuesto

La tabla 5 muestra la prueba de promedios de Duncan para las variables de desarrollo vegetativo (altura de planta, diámetro de copa, diámetro de tallo) y las variables de rendimiento (longitud de fruto, peso de frutos por planta y rendimiento por hectárea) para la interacción entre la aplicación de gallinaza descompuesta y el aserrín descompuesto en el demostrándose que la aplicación de $3 \mathrm{~kg}$ de humus de lombriz/planta, mejoró las características agronómicas evaluadas e incrementó el rendimiento con $3863.8 \mathrm{~kg} / \mathrm{ha}$ de fruta, siendo este rendimiento inferior a lo obtenido en esta investigación. 
orgánicos y las aplicaciones de aserrín descompuesto solas y en combinación, generaron los promedios más bajos en todas las variables estudiadas. Al respecto, Riva (2019) indica que el abonamiento orgánico se incorpora después del poseado en forma localizada, al momento del transplante de las plantas a campo definitivo, empleando $2 \mathrm{~kg}$ de gallinaza descompuesta, sin embargo, en el presente estudio se utilizó 3 y $6 \mathrm{~kg}$ de gallinaza descompuesta obteniéndose en estos tratamientos los mejores promedios en todas las variables evaluadas incluido el rendimiento por hectárea. Asimismo, Paredes (2017) estudio cuatro densidades de siembra en el rendimiento del cultivo de ají charapita (Capsicum frutescens L.) bajo las condiciones agroecológicas de la provincia de Tocache, obteniendo el mayor peso de frutos en el distanciamiento $0,9 \mathrm{~m} \quad \mathrm{x} \quad 0,7 \mathrm{~m}$, con un rendimiento de $2556,25 \mathrm{~kg} / \mathrm{ha}$, siendo estos resultados inferiores a los obtenidos en este estudio. Estrada (2005) indica que la utilidad de la gallinaza procesada y correctamente descompuesta, aporta al suelo materia orgánica, con lo cual aumenta su capacidad de retención de agua, es una fuente muy rica en elementos nutritivos para las plantas, sumándose su importancia como forma de reciclaje natural y por su bajo costo.

Tabla 5

Variables de desarrollo vegetativo y rendimiento para la interacción entre el factor gallinaza descompuesta y el aserrín descompuesto, a los 183 días de instalado en campo definitivo

\begin{tabular}{llcccccccc}
\hline $\begin{array}{c}\text { Gallinaza } \\
\text { descomp. }\end{array}$ & $\begin{array}{c}\text { Aserrín } \\
\text { descomp. }\end{array}$ & $\begin{array}{c}\text { Alt. } \\
\text { planta } \\
\text { (cm) }\end{array}$ & $\begin{array}{c}\text { Diám. } \\
\text { copa }(\mathbf{c m})\end{array}$ & $\begin{array}{c}\text { Diám. } \\
\text { tallo } \\
\text { (cm) }\end{array}$ & $\begin{array}{c}\text { Diám. } \\
\text { fruto } \\
(\mathbf{c m})\end{array}$ & $\begin{array}{c}\text { Long. } \\
\text { fruto } \\
(\mathbf{c m})\end{array}$ & $\begin{array}{c}\text { Peso } \\
\text { frutos/ } \\
\text { planta } \\
\text { (kg) }\end{array}$ & $\begin{array}{c}\text { Rend. } \\
\text { (kg/ha) }\end{array}$ \\
\hline $0 \mathrm{~kg} /$ planta & $0 \mathrm{~kg} /$ planta & $75.50 \mathrm{~cd}$ & $93.66 \mathrm{~cd}$ & $1.68 \mathrm{c}$ & $1.20 \mathrm{~d}$ & $0.99 \mathrm{f}$ & $2.783 \mathrm{c}$ & $18558 \mathrm{c}$ \\
$0 \mathrm{~kg} /$ planta & $3 \mathrm{~kg} /$ planta & $77.41 \mathrm{~cd}$ & $87.91 \mathrm{~d}$ & $1.75 \mathrm{bc}$ & $1.25 \mathrm{~d}$ & $1.06 \mathrm{de}$ & $2.635 \mathrm{c}$ & $17569 \mathrm{c}$ \\
$0 \mathrm{~kg} /$ planta & $6 \mathrm{~kg} /$ planta & $69.83 \mathrm{~d}$ & $89.33 \mathrm{~d}$ & $1.70 \mathrm{c}$ & $1.21 \mathrm{~d}$ & $1.03 \mathrm{ef}$ & $3.033 \mathrm{~b}$ & $20222 \mathrm{~b}$ \\
$3 \mathrm{~kg} /$ planta & $0 \mathrm{~kg} /$ planta & $91.50 \mathrm{~b}$ & $121.08 \mathrm{ab}$ & $1.83 \mathrm{~b}$ & $1.32 \mathrm{bc}$ & $1.09 \mathrm{cde}$ & $4.550 \mathrm{a}$ & $30333 \mathrm{a}$ \\
$3 \mathrm{~kg} /$ planta & $3 \mathrm{~kg} /$ planta & $80.58 \mathrm{~cd}$ & $92.91 \mathrm{~cd}$ & $1.73 \mathrm{c}$ & $1.21 \mathrm{~d}$ & $1.04 \mathrm{ef}$ & $3.433 \mathrm{~b}$ & $22888 \mathrm{~b}$ \\
$3 \mathrm{~kg} /$ planta & $6 \mathrm{~kg} /$ planta & $79.75 \mathrm{~cd}$ & $100.33 \mathrm{~cd}$ & $1.70 \mathrm{c}$ & $1.31 \mathrm{c}$ & $1.11 \mathrm{bcd}$ & $3.525 \mathrm{~b}$ & $23499 \mathrm{~b}$ \\
$6 \mathrm{~kg} /$ planta & $0 \mathrm{~kg} /$ planta & $110.08 \mathrm{a}$ & $129.25 \mathrm{a}$ & $1.95 \mathrm{a}$ & $1.42 \mathrm{a}$ & $1.20 \mathrm{a}$ & $4.733 \mathrm{a}$ & $31555 \mathrm{a}$ \\
$6 \mathrm{~kg} /$ planta & $3 \mathrm{~kg} /$ planta & $74.50 \mathrm{~cd}$ & $93.91 \mathrm{~cd}$ & $1.71 \mathrm{c}$ & $1.34 \mathrm{bc}$ & $1.15 \mathrm{abc}$ & $3.508 \mathrm{~b}$ & $23388 \mathrm{~b}$ \\
$6 \mathrm{~kg} /$ planta & $6 \mathrm{~kg} /$ planta & $82.75 \mathrm{bc}$ & $107.66 \mathrm{bc}$ & $1.76 \mathrm{bc}$ & $1.37 \mathrm{ab}$ & $1.17 \mathrm{ab}$ & $4.508 \mathrm{a}$ & $30055 \mathrm{a}$ \\
\hline
\end{tabular}

*Letras iguales no presentan diferencias significativas. Duncan $\mathrm{p} \leq 0,05$ 


\section{Conclusión}

Al aumentar la cantidad de gallinaza descompuesta aplicada a la planta, aumentan los valores en las variables de desarrollo vegetativo y de rendimiento, siendo la aplicación de $6 \mathrm{~kg}$ de gallinaza por planta la que logró los mejores promedios en todas las variables estudiadas, resaltándose el rendimiento por hectárea logrado por este tratamiento $(28333 \mathrm{~kg} / \mathrm{ha})$.

A medida que se aumenta la cantidad de aserrín descompuesto aplicada a la planta, los valores en todas las variables estudiadas disminuyen, siendo el tratamiento sin aplicación de aserrín descompuesto el que logró los mejores promedios incluyendo el rendimiento por hectárea $(26816 \mathrm{~kg} / \mathrm{ha})$, corroborándose este comportamiento en la interacción de los factores, en los cuales la aplicación de $6 \mathrm{~kg}$ de gallinaza descompuesta sola logró los mejores promedios en todas las variables estudiadas incluyendo en rendimiento por hectárea $(31555 \mathrm{~kg} / \mathrm{ha})$.

\section{Referencia bibliográfica}

Arévalo Mogrovejo, M, (2012). Evaluación del rendimiento en 5 densidades de siembra de Capsicum frutescens L. (Aji Charapita) en un Ultisols de
Pucallpa. Tesis para optar el título de Ingeniero Agrónomo. Universidad Nacional de Ucayali. Facultad de Ciencias Agropecuarias. Pucallpa, Perú. Disponible en: http://repositorio.unu.edu.pe/bitstrea m/handle/UNU/1521/000001942T.pd $\mathrm{f}$ ? sequence $=1$ \&isAllowed $=\mathrm{y}$

Abreu Cruz, E., Araujo Camacho, E., Rodríguez Jimenez, S. L., Valdivia Ávila, A. L., Fuentes Alfonso, L., Pérez Hernández, Y. (2018). Efecto de la aplicación combinada de fertilizante químico y humus de lombriz en Capsicum annuum. Revista Centro Agrícola Vol.45, No.1, enero-marzo, 52-61, 2018. Disponible en: http://cagricola.uclv.edu.cu/descargas /pdf/V45-Numero_1/cag07118.pdf

Cabaleiro, F. A., Sainz, M. J., SeoaneLabandeira, S., López-Mosquera, M. E. (2018). Efectos en suelo y fruto de la fertilización de pimiento con estiércol de pollo peletizado. Recursos Rurais (2017) $\mathrm{n}^{\mathrm{o}}$ 13: 47-53.

Estrada Pareja, M. M. (2005). Manejo y procesamiento de la gallinaza. Revista Lasallista de Investigación, vol. 2, núm. 1, enero-junio, 2005, pp. 43-48. Corporación Universitaria Lasallista. 
Antioquia, Colombia. Disponible en: https://www.redalyc.org/pdf/695/695 20108.pdf

García, A. (2011). Estudio de Mercado de Variedades Sub-utilizadas de Ajíes Nativos (Capsicum spp) en el Perú. Tesis de máster en marketing. Universidad Peruana de Ciencias Aplicadas. Escuela de Postgrados. 2011. $211 \mathrm{p}$.

Garcia Yi， J. (2013). Segmentación del mercado de ajíes nativos subutilizados mediante modelos Poisson y Probit ordenados en Lima, Agroalimentaria, (19).

http://erevistas.saber.ula.ve/index.php /agroalimentaria/article/view/5274/50 66

López Lituma, L. E. (2015). Biol y gallinaza en la producción del ají tabasco (Capsicum annum) en la zona de Patricia Pilar. Tesis para la obtención del título de Ingeniero Agropecuario. Universidad Técnica Estatal de Quevedo. Carrera Ingeniería Agropecuaria. Quevedo, Ecuador. Disponible en: https://repositorio.uteq.edu.ec/bitstrea m/43000/1508/1/T-UTEQ-0171.pdf
Lozano Sánchez, M. (2016). Efecto de cuatro dosis de materia orgánica (pollaza) en el cultivo de ají pimentón (Capsicum annuит L) variedad California Wonder en el distrito de Lamas. Tesis para optar el título de Ingeniero Agrónomo. Universidad Nacional de San Martín. Facultad de Ciencias Agrarias. Tarapoto, Perú. Disponible en:

https://core.ac.uk/download/pdf/2873 29342.pdf

Miranda Ruiz, E., Rengifo Rivadeneira, G. R. (2016). Efecto de dos tipos de abonos orgánicos en el cultivo de ají charapita (Capsicum frutescens), distrito de Manantay provincia de Coronel Portillo-Ucayali. Rev. Tzhoecoen Edición Vol. 8 / N 02, ISSN 19973985

Masaquiza Chimbolema, M. F. (2016). Influencia del abono orgánico biol, sobre el comportamiento agronómico y productividad del cultivo de pimiento (Capsicum annuum L.), en El Cantón Cumandá provincia de Chimborazo. Tesis como requisito para obtener el grado de Ingeniero Agropecuario. Universidad Técnica de Ambato. Facultad de Ciencias 
Agropecuarias. Carrera de Ingeniería

Agropecuaria. Disponible en:

https://repositorio.uta.edu.ec/bitstrea

$\mathrm{m} / 123456789 / 24083 / 1 /$ tesis\%20004

\%20Ingenier\%C3\%ADa\%20Agropec

uaria\%20-

\%20Maria\%20Fernanda\%20Masaqq

uisa\%20-\%20cd\%20004.pdf

Mozombite García, J. L. (2012). Efecto de diferentes niveles de aplicación orgánica (humus de lombriz) en la producción de ají charapita (Capsicum frutescens) en un suelo Ultisol de Pucallpa. Tesis para optar el título de Ingeniero Agrónomo. Universidad Nacional de Ucayali. Facultad de Ciencias Agropecuarias. Ucayali, Perú. Disponible en: http://repositorio.unu.edu.pe/bitstrea m/handle/UNU/1869/000000740T.p df? sequence $=1$ \&isAllowed $=y$

Ocupa Laban, M. E. (2019). Efecto de dos dosis de biofertilizante (biol) de preparación artesanal en el rendimiento de ají paprika morron (Capsicum annuum L.) en la localidad de Marcavelica Sullana Piura. Tesis para para optar el título de Ingeniero Agrónomo. Universidad Nacional de Piura. Facultad de
Agronomía. Piura, Perú. Disponible

en:

https://repositorio.unp.edu.pe/bitstrea m/handle/UNP/1949/AGR-OCU-

LAB-

2019.pdf? sequence $=1 \&$ isAllowed $=y$

Paredes Bautista, J. J. P. (2017). Evaluación de cuatro densidades de siembra en el rendimiento del cultivo de ají charapita (Capsicum frutescens L.) en el sector Almendras, provincia de Tocache. Tesis para optar el título profesional de Ingeniero Agrónomo. Universidad Nacional de San Martín. Facultad de Ciencias Agrarias. Escuela Profesional de Agronomía. Tarapoto, Perú. Disponible en: http://repositorio.unsm.edu.pe/bitstre am/handle/11458/3367/AGRONOM $\% \mathrm{C} 3 \% 8 \mathrm{DA} \% 20-$ \%20Jhonathan\%20Jean\%20Pierre\% 20Paredes\%20Bautista.pdf?sequence $=1 \&$ isAllowed $=\mathrm{y}$

Reyes Pérez, J. J., Luna Murillo, R. A., Reyes Bermeo, M. Del R., Zambrano Burgos, D., Vázquez Morán, V. F. (2017). Fertilización con abonos orgánicos en el pimiento (Capsicum annuит L.) y su impacto en el rendimiento y sus componentes. 
Revista Centro Agrícola Vol.44, No.4, octubre-diciembre, 88-94, 2017.

Disponible

en:

http://scielo.sld.cu/pdf/cag/v44n4/cag 13417.pdf

Riva Ruiz, R. (2019). Manual del cultivo de ají charapita (Capsicum frutescens L.). Primera edición. Imprenta EZAAC S.R.L. ISBN: 978-9972-2856-4-6. Universidad Nacional de Ucayali. Ucayali, Perú. Disponible en: http://repositorio.unu.edu.pe/bitstrea m/handle/UNU/4008/UNU_AGRON OMIA_2019_L_RITARIVARUIZ.pd f? sequence $=1 \&$ isAllowed $=\mathrm{y}$

Velásquez Ramírez, M. G. (2016). Experimentación con fertilizantes foliares provenientes del reciclaje de residuos orgánicos en ají amarillo (Capsicum baccatum L.var pendulum) aplicando herramientas participativas. Tesis para optar el título de Ingeniero Agrónomo. Universidad Nacional Agraria La Molina. Facultad de Agronomía. Lima, Perú. Disponible en:

https://repositorio.lamolina.edu.pe/bit stream/handle/UNALM/1962/F04-

V447-

T.pdf?sequence $=1 \&$ isAllowed $=y$ 